\title{
Personalized medicine: A transformative approach is needed
}

\author{
Thomas J. Hudson MD
}

Published at www.cmaj.ca on Feb. 18, 2009.

$\mathrm{T}$ he sequencing of the human genome has paved the way for discoveries that are providing new insight into the causes of human diseases. The medical application of this knowledge to individuals is ushering in a new era of personalized medicine. Translating this into benefits for patients and society will create challenges and opportunities for biomedical research, the medical profession and the health care system.

In the 7 years since the first draft of the human genome sequence was released, ${ }^{1}$ there has been an explosion in knowledge about the genetic causes of common and rare diseases. ${ }^{2,3}$ New technologies allow rapid, comprehensive and accurate measures of genes and proteins in blood and other tissues, leading to more accurate diagnoses and new ways of predicting adverse effects of drugs. This knowledge is disseminating into clinical investigative disciplines such as pathology, radiology and nuclear medicine. Drugdevelopment programs in biotechnology and pharmaceutical companies are rapidly moving toward a "target-based" approach as a result of the increasingly precise molecular understanding of diseases. An example of a cancer gene target that has been used as a molecular marker is HER2/neu, which encodes the protein targeted by the therapeutic antibody trastuzumab. ${ }^{4}$ Considerable research is underway to identify additional target genes and proteins involved in many human diseases, with the hope that targeted therapies will prove more effective and less likely to affect normal tissues (i.e., cause unnecessary adverse outcomes). Genetic testing companies are multiplying, and some are capitalizing on the appetite of consumers to "know" more about their risk of developing diseases, irrespective of the predictive accuracy or inaccuracy of the test.

There is deep concern that too few discoveries will benefit Canadian patients, unless there are transformative changes in biomedical research and health care delivery that enable quick assessment of a rapidly growing number of new diagnostics and therapeutics, clear guidelines regarding usage in medical care, enabling policies, and appropriate levels of reimbursement. Commercial opportunities arising from Canadian laboratories in academia and biotechnology companies are at stake if funding and other support mechanisms are not competitive with those in other jurisdictions. All the stakeholders, funding agencies, researchers, clinicians, health service providers and governments appreciate the challenges, but no one seems to have a full grasp of what is needed to pave the way for personalized medicine. No one can be blamed for the exponential burst of genome-driven applications. The genomic revolution is happening too quickly! Let's not panic this is a good problem!

\section{Key points}

- Advances in human genome research are leading health care toward a new era in personalized medicine.

- Moving discoveries to the clinic will be a challenge that needs to be addressed at many levels, including clinical research, commercialization, policy changes and education.

- Key stakeholders in Canada need to create an alliance that can identify challenges and opportunities, and implement a strategy that will introduce personalized medicine safely and efficiently into the health care system.

\section{Waves of translation}

There are many outstanding opportunities in the short term that should not be missed. Canada has an outstanding research community involved in international projects that have described the complexity of the variation in the human genome ${ }^{5,6}$ and that have developed methods to study this variation in medical research. In conjunction with other international discoveries, ${ }^{3}$ Canadians have been involved in the first wave of translation leading to personalized medicine: discovery of genes, mutations, biomarkers and molecular pathways that are associated with diseases such as autism, ${ }^{7}$ type 2 diabetes, ${ }^{8}$ coronary artery disease, ${ }^{9}$ inflammatory bowel disease, ${ }^{10-12}$ susceptibility to infection, ${ }^{13}$ colon cancer ${ }^{14}$ and drug response. ${ }^{15}$ Canadian scientists have been successful in engaging multiple partners within Canada and beyond, including large multinational companies with funding to support large-scale genome-style research projects (e.g., the Structural Genomics Consortium). More discovery research that is driven by a powerful new generation of genome technologies is needed. These technologies make it possible to conduct the comprehensive and high-resolution genome analyses that are needed for the study of large numbers of disease genes in numerous patients with these diseases. New international disease genome projects, such as the International Cancer Genome Consortium, are underway and will generate data sets that are more than a 1000-fold larger than the original sequence obtained by the Human Genome Project. Canadian scientists cannot lag behind after leaping ahead and securing prominent positions on international podiums.

To launch the second wave of translation activity from the Human Genome Project to personalized medicine, major invest-

Thomas Hudson is President and Scientific Director of the Ontario Institute for Cancer Research, Toronto, Ont. He is also a professor in the Departments of Molecular Genetics and Medical Biophysics, Faculty of Medicine, University of Toronto, Toronto, Ont. 
ment is needed in clinical research to integrate new genomic knowledge in clinical trials and population research. Important challenges need to be addressed, including a paucity of clinician researchers, particularly in disciplines such as molecular pathology and imaging, hesitancy in moving toward larger and more complex clinical trials that include testing diagnostic markers in combination with drugs, and inadequate funding from funding agencies and industry of these "high-content" trials. The increasing complexity of regulatory approval hinders the use of new trial designs, even if these would provide important information about responders, nonresponders and individuals at high risk for adverse outcomes. It is exciting to note that after a decade of planning workshops, Canada is finally launching large population studies, such as the Canadian Partnership for Tomorrow Project, that are well coordinated with international cohorts, such as the Public Population Project in Genomics. These critical resources will need long-term support to realize their potential for understanding the effects of genes and environment in disease predisposition.

The third wave of translational activity involves education, communication, health services research, policy-making and adoption by health care providers. The complex nature of personalized medicine will necessarily require changes in these areas. Molecular pathology laboratories are struggling to provide quality results for the first wave of gene-based tests, such as $H E R 2 /$ neu testing for breast cancer and KRAS testing for colorectal cancer, ${ }^{16}$ that are required before starting therapies that have significant benefits in specific subsets of patients. How will these laboratories cope with hundreds, if not thousands, of tests if not by centralization of molecular testing services, automation, adoption of genomic technologies, databases and training? Who will advise health insurance boards on which tests to support? Who will have the expertise to say if a $\$ 100$ test is required for all patients considered for an expensive drug before initiating therapy? How should new diagnostic companies be supported to gain access and compete in Canadian and international markets? How should these companies be regulated?

\section{Partnership for action}

Where do we start? Let's review the mechanisms that support research in personalized medicine. Let's identify the funding gaps. Let's take a hard look at the barriers between agencies. Let's recognize that no funding agency has a monopoly on ideas, mandates or the research community. Let's look at the barriers to translation between basic and clinical sciences. Let's create synergies between agencies that fund technology platforms, clinical research, infrastructure and training. Let's create funding envelopes dedicated to personalized medicine, including discovery, clinical research and commercialization. Given that these goals need to be developed coherently and inclusively, let's create a partnership of stakeholders in academia, governments, foundations and companies. The Personalized Medicine Coalition, based in Washington, DC, is a model to consider. Canada may have opportunities to develop unique approaches to personalized medicine, given its universal health care coverage, international leadership in human genetic research, high participation rates in clinical trials and access to computerized provincial health databases for evaluation of safety and effectiveness of therapeutic interventions. We have opportunities to become leaders in this new industry. Let's think outside the box to capitalize on Canadian strengths and provide a model that may benefit other nations.

Should we wait for an economic recovery in Canada before we start addressing this problem? No. The consequences of chronic disorders for individuals, their families and society are enormous. In less than a decade, genomics has yielded important new insight into the causes and mechanisms of chronic disorders and drug response that point to the undisputed fact that most of the diagnostic categories that we currently use in medicine classify patients into clusters that overlook the fact that individual patients have different diseases and responses to therapies. There clearly is a benefit to research in personalized medicine: prevention and screening strategies targeting high-risk individuals, avoidance of serious adverse outcomes, and better matching of therapies to disease and individual profiles. If we are slow to act, there will be economic consequences because we will lose opportunities for the development and commercialization of new products and for the creation of new jobs in Canada. Canada will also fail to benefit from a reduction in the staggering costs because of the loss of productivity caused by illness.

Competing interests: Thomas Hudson is affiliated with several projects discussed in this article. He is cofounder of the International Cancer Genome Consortium and the Public Population Project in Genomics. Thomas Hudson is president and scientific director of the Ontario Institute for Cancer Research, which is funded by the Government of Ontario through the Ministry of Research and Innovation. He currently receives research funds from Genome Canada, the Canadian Cancer Society, the European Commission and the US National Institutes of Health. He is a past recipient of salary or research awards from the Canadian Institutes of Health Research, the Canada Foundation for Innovation, and the Networks of Centres of Excellence. Thomas Hudson has also been vice-chair of the Board of Directors for Genome Canada (2005-2008) and a member of the Institute Advisory Board, Canadian Institutes of Health Research Institute of Genetics (2001-2006).

\section{REFERENCES}

1. Lander ES, Linton LM, Birren B, et al. Initial sequencing and analysis of the human genome. Nature 2001;409:860-921.

2. Pennisi E. Breakthrough of the year. Human genetic variation. Science 2007:318:1842-3.

3. Manolio TA, Brooks LD, Collins FS. A HapMap harvest of insights into the genetics of common disease. J Clin Invest 2008;118:1590-605.

4. Slamon DJ, Leyland-Jones B, Shak S, et al. Use of chemotherapy plus a monoclonal antibody against HER2 for metastatic breast cancer that overexpresses HER2. N Engl J Med 2001;344:783-92.

5. International HapMap Consortium. A haplotype map of the human genome. $\mathrm{Na}$ ture 2005;437:1299-320.

6. Redon R, Ishikawa S, Fitch KR, et al. Global variation in copy number in the human genome. Nature 2006;444:444-54.

7. Szatmari P, Paterson AD, Zwaigenbaum L, et al.; Autism Genome Project Consortium. Mapping autism risk loci using genetic linkage and chromosomal rearrangements. Nat Genet 2007;39:319-28. 
8. Sladek R, Rocheleau G, Rung J, et al. A genome-wide association study identifies novel risk loci for type 2 diabetes. Nature 2007;445:881-5.

9. McPherson R, Pertsemlidis A, Kavaslar N, et al. A common allele on chromosome 9 associated with coronary heart disease. Science 2007;316:1488-91.

10. Rioux JD, Xavier RJ, Taylor KD, et al. Genome-wide association study identifies new susceptibility loci for Crohn disease and implicates autophagy in disease pathogenesis. Nat Genet 2007;39:596-604.

11. Silverberg MS, Cho JH, Rioux JD, et al. Ulcerative colitis-risk loci on chromosomes $1 \mathrm{p} 36$ and 12q15 found by genome-wide association study. Nat Genet 2009;41:216-20.

12. Villani AC, Lemire M, Fortin G, et al. Common variants in the NLRP3 region contribute to Crohn's disease susceptibility. Nat Genet 2009;41:71-6.

13. Mira MT, Alcaïs A, Nguyen VT, et al. Susceptibility to leprosy is associated with PARK2 and PACRG. Nature 2004;427:636-40.

14. Zanke BW, Greenwood CM, Rangrej J, et al. Genome-wide association scan identifies a colorectal cancer susceptibility locus on chromosome 8q24. Nat Genet 2007;39:989-94.

15. Zhu CQ, da Cunha Santos G, Ding K, et al. Role of KRAS and EGFR as biomarkers of response to erlotinib in National Cancer Institute of Canada Clinical Trials Group Study BR.21. J Clin Oncol 2008;26:4268-75.

16. Molecular Oncology Task Force. Ensuring access to high quality molecular oncology laboratory testing and clinical cancer genetic services in Ontario. Toronto (ON): Cancer Care Ontario; 2008. Available: www.cancercare.on.ca/documents /MolecOncReport.pdf (accessed 2009 Feb. 12).

Correspondence to: Dr. Thomas J. Hudson, MaRS Centre, South Tower, 101 College St., Ste. 800, Toronto ON M5G 0A3, tom.hudson@oicr.on.ca

\section{Additional resources}

Canada Foundation for Innovation

- www.innovation.cal

Canadian Institutes of Health Research

- www.cihr-irsc.gc.ca/e/193.html

Canadian Partnership for Tomorrow Project

- www.partnershipagainstcancer.ca/tomorrow

Human Genome Project

- www.genome.gov/10001772

Structural Genomics Consortium

- www.thesgc.com/

International Cancer Genome Consortium

- www.icgc.org/

Ontario Institute for Cancer Research

- www.oicr.on.ca

Networks of Centres of Excellence

- www.nce.gc.cal

Personalized Medicine Coalition

- www.personalizedmedicinecoalition.org/

Public Population Project in Genomics

- www.p3gconsortium.org/ 